\title{
Design of a Broadband Zig-Zag Pyramidal Log-Periodic Antenna
}

\author{
Wendy L. Lippincott \\ Naval Research Lab, Wash., DC, 20375, http://www.nrl.navy.mil
}

A pyramidal zig-zag toothed log-periodic (LP) antenna with a customized cable quarter-wave transformer was developed as a dual-linear high-power feed for a large 60 foot diameter reflector with $\mathrm{f} / \mathrm{D}$ of 0.49 . The pyramidal design was chosen over a crossed log-periodic pole design since it provides matching $\mathrm{E}$ - and $\mathrm{H}$-plane patterns (the broader $\mathrm{H}$-plane is made narrower with the two opposing elements acting as an array in that dimension). Three separate antennas with frequency ranges of $0.2-0.5,0.5-1$, and $1-2 \mathrm{GHz}$ were built.

Log-periodic antennas have well-known design parameters and are frequently designed with a trapezoidal tooth structure (DuHamel, R.H., \& Ore, F.R., IRE Nat. Conv. Record, pp. 128-137, 1959). ARA Inc. markets such an antenna. The zig-zag design used herein (Fig. 1) is similar to a CP pyramidal LP design produced by Microstar Inc. but adds the transformer. A customized preprocessing code was used to generate input for the FEKO code method of moments model using a variable gridding (smaller mesh at the top) to conserve memory requirements and processing speed. Parameters of inner, outer, and pyramidal angles, and teeth proportional design ratio, $\tau$, are used to optimize the pattern for the reflector (optimally a $10 \mathrm{~dB}$ edge taper).

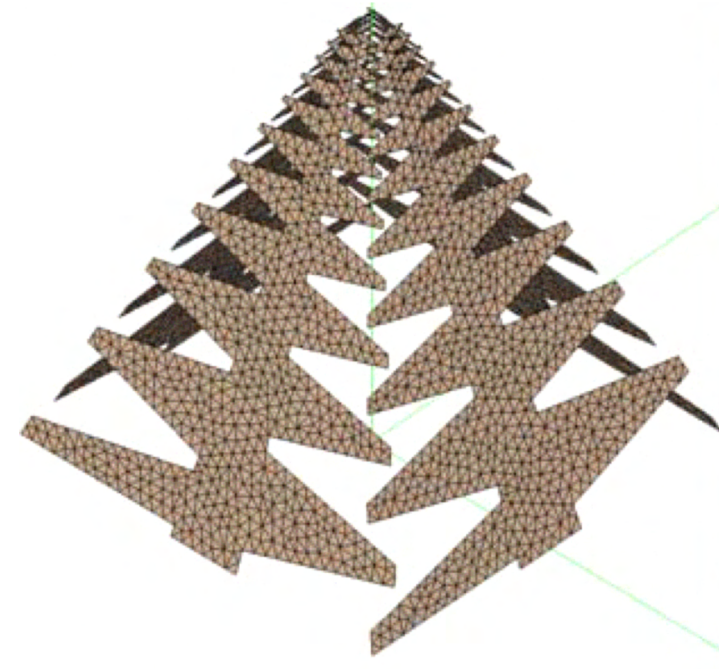

(a)

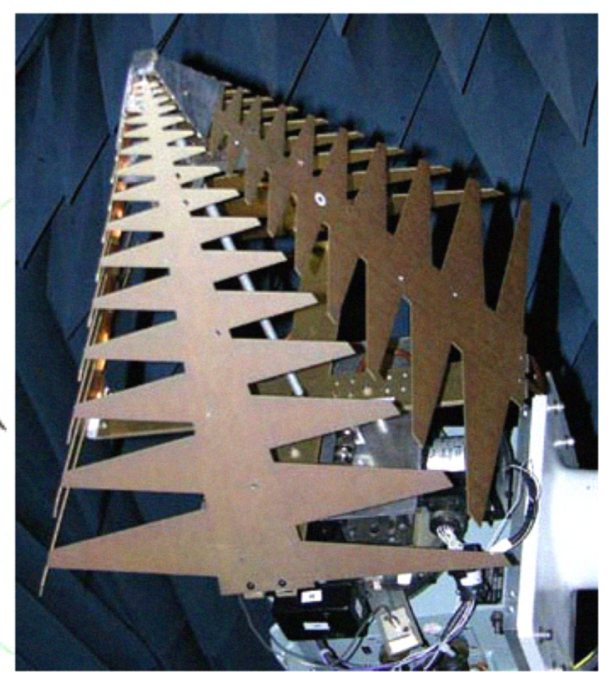

(b)

Fig. 1 Pyramidal Log-Periodic 'Zig-Zag' Antenna (a) FEKO model (b) photo

The antenna is fed by two low-loss semi-rigid cables running up the inside center of two of the LP sides. The outer conductor is attached to the side plates, and the inner cable is unsheathed and attached to the opposing side plate at the top. The smaller 1-2 GHz LP plates were machined out of brass so that the cable could be 
soldered to the side plate and provide good contact and impedance matching. For the larger LPs, gold-irridited Al was substituted for brass to reduce weight. The cables were then clamped onto the sides since copper cannot solder to Al. Matching 'dummy' cables are attached to the inside non-fed sides of the LP.

Center support pieces of the antenna were cross-bars machined of Lexan plastic (Fig. 2). A $1 / 2$ " threaded rod roughly $3 / 4$ of the LP height was attached at the base and the plastic supports held in place with bolts on each side. The LP side pieces were screwed into these supports. A small plastic inset piece at the top was screwed into the sides with plastic screws to further anchor the antenna and prevent the solder joint of the top cable from breaking. All designs of the LP sides, bases, and center support pieces were easily generated by inputting $\mathrm{x}, \mathrm{y}, \mathrm{z}$ data into a simple CAD program, extruding it, and converting it to a .igs file. This file was sent to a sheet metal manufacturer (Prototech) who produced the parts.

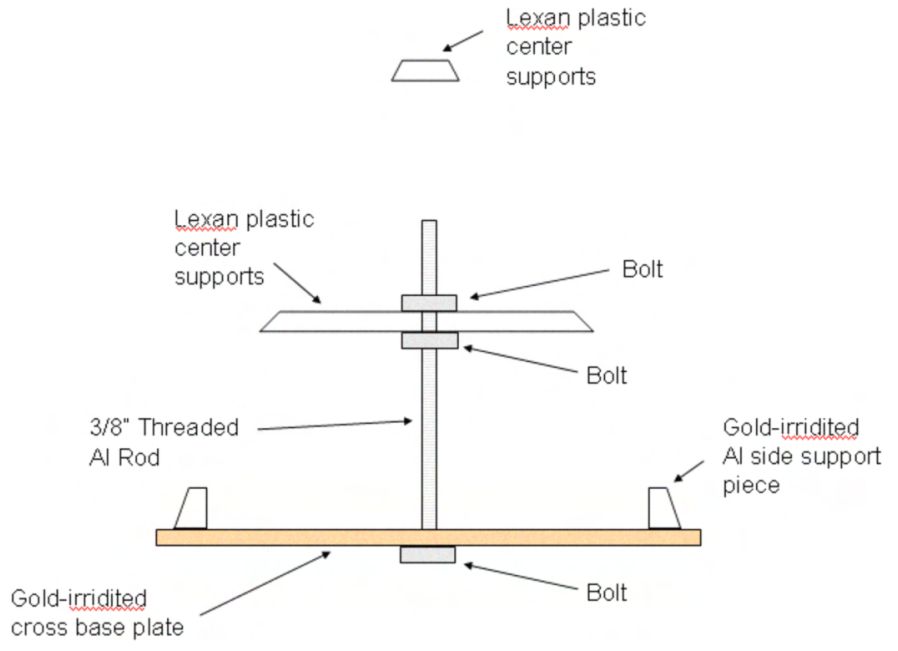

Fig. 2 Support Structure for the LPs

A quarter-wave transformer was built into the end of the cable to reduce the VSWR to below 2:1 (particularly necessary due to the high power of $1 \mathrm{~kW}$ the antennas are to be used at). This type of antenna is often built for circular polarization (CP) with a hybrid feeding the cables. The hybrid drastically improves the VSWR (effectively shorting out the reflected power), often eliminating the need for a matching transformer.

The 0.25" diameter cable was low-loss and could handle more power than a standard PTFE cable (up to $1.8 \mathrm{~kW}$ of $\mathrm{CW}$ power at $1 \mathrm{GHz}$ and $1.25 \mathrm{~kW}$ at 2 $\mathrm{GHz}$ ). The $\lambda / 4$ transformer was matched to below the center frequency of the band (ex. 1.4 GHz for the 1-2 GHz LP) because the low end of the band did not respond to the added tuning plates at the top and so was more critical to match with the transformer.

The transformer was built by taking the outer conductor and dielectric off the cable for the $\lambda / 4$ distance plus the length of the wire stub (for the $0.5-1 \mathrm{GHz}$ 
antenna this is $4.2 "+0.8$ "). The inner cable is then dipped in a Nitric/Sulfuric acid bath until it is reduced in size to 0.061 " diameter. The inner diameter of the outer conductor is 0.21 ". Using a simple free application like AppCAD from Agilent, this corresponds to a $77 \Omega$ impedance (with an air dielectric) which is the square root of the products of the inherent impedances of the LP (120 $\Omega$ ) and the cable $(50 \Omega)$. The outer conductor is then replaced onto the wire with Teflon spacers (constructed from the dielectric of another wire) and the outer conductors soldered together. Fig. 3 illustrates this process.

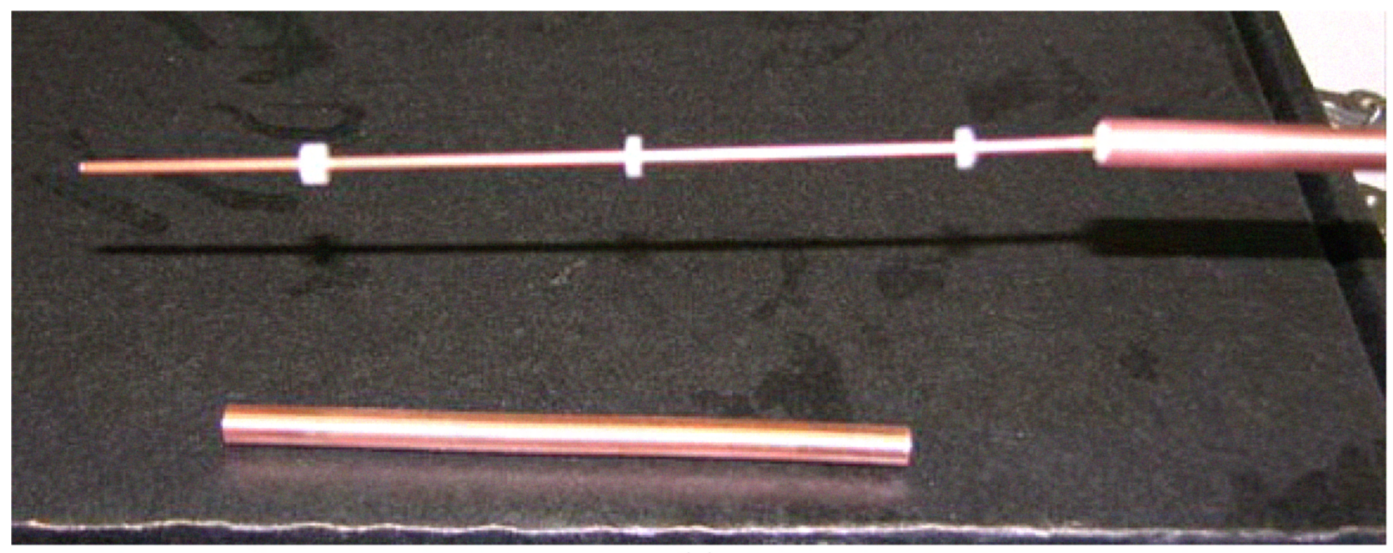

(a)

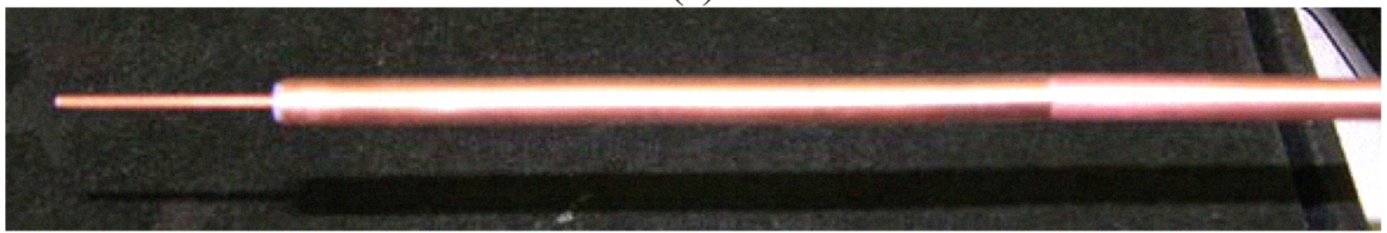

(b)

Fig. 3 Quarter-wave transformer (a) under construction showing teflon spacers from another cable and (b) completed design

For the 1-2 GHz brass LP, soldering the cable onto the brass proved difficult because too much applied heat melts the cable dielectric and can short out the cable. However, keeping a good connection at the top of the LP was critical and could only be obtained with solder. To solve this problem, the outer conductor of the transformer section was soldered onto the LP side first and then the rest of the cable was put on and clamped. The 'dummy' cables on the non-fed sides of the LP were soldered since their electrical integrity was not essential.

The LPs were sized considerably larger than the lowest frequency halfwavelength to reduce the reflections and lower the VSWR at the low end of the band. For example: for the 0.2 to $0.5 \mathrm{LP}$, the $\lambda / 2$ radiating width for $0.2 \mathrm{GHz}$ is 29.5 ", but the necessary width at the base of the pyramid is 42 ". The top end of the LP was sized small (ex. 1.2" spacing for 0.2 to $0.5 \mathrm{LP}$ ) mainly to avoid a long cable element between the sides. The LP non-fed sides were isolated electrically from the ground by adding plastic strips where the sides met the $\mathrm{Al}$ base support. 
The $\lambda / 4$ transformer reduces the VSWR but also inherently reduces the bandwidth of the antenna. Tuning plates screwed onto the top area of the LP were used to further lower the VSWR (see Fig. 1). These acted to suppress some of the higher order modes and to provide better impedance matching. The tuning plates varied slightly from antenna to antenna due to slight differences in the spacing of the sides. This side spacing was difficult to accurately maintain because screwing the LP sides into the plastic center support pieces consistently was difficult. Further mechanical development would improve this aspect of the design.

Sample patterns and VSWR are shown in Figs. $4 \& 5$. Peak gain was flat and varied at most $2 \mathrm{~dB}$ across the bands. Cross-polarization (Cross-pol) varied greatly but was typically at $15 \mathrm{~dB}$ down from the peak gain at 0 deg., slightly higher than the $18 \mathrm{~dB}$ predicted with the FEKO code.

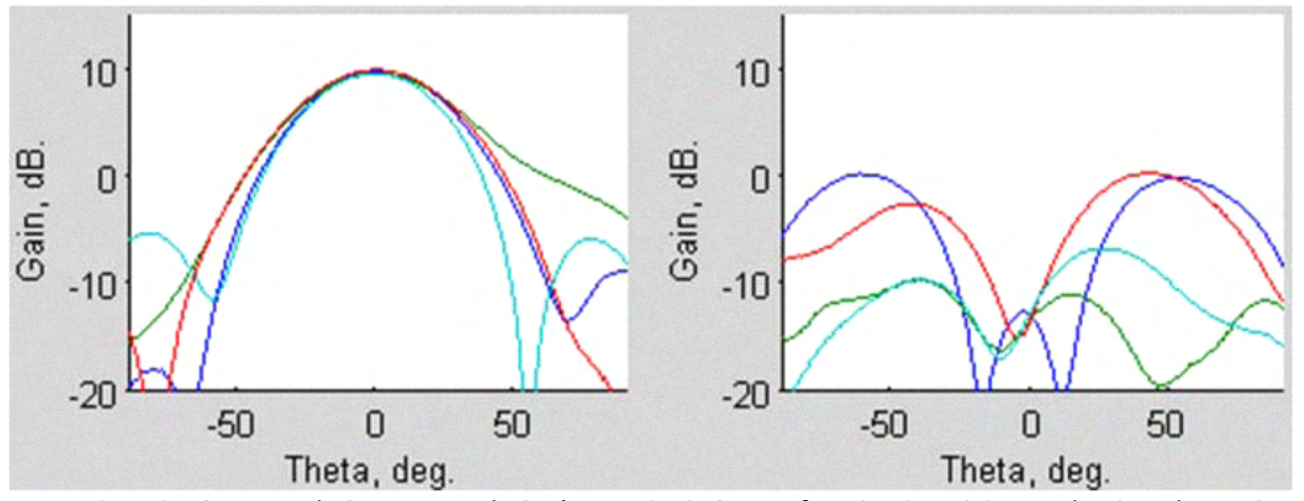

Fig. 4 Co- and Cross-Pol Gain at $0.6 \mathrm{GHz}$ for $0,45,90$, and $135 \mathrm{deg}$. Cuts

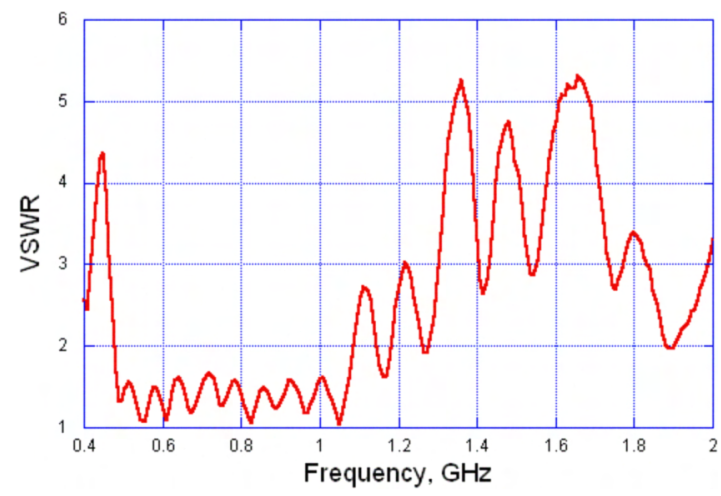

Fig. 5 VSWR for $0.5-1 \mathrm{GHz}$ LP

The FEKO simulations showed that slight variations in the design parameters made little difference to the gain flatness, cross-polarization, and VSWR. The key factors for these were keeping the LP sides perfectly orthogonal to one another (pyramidal) and also maintaining good contact of the cables to the LP sides at the top of the pyramid either with solder or clamps. The LP radiates at the $\lambda / 2$ wavelength width for any given frequency. This phase center movement causes losses when the LP is used as a reflector feed. 